\title{
Fast Identification of Active and Reactive Current Component for Single Phase Grid Interconnection
}

\author{
Samir Gautam*, Dylan Dah-Chuan $\mathrm{Lu}^{\dagger}$,Yuezhu $\mathrm{Lu}^{*}$, and Weidong Xiao* \\ ${ }^{*}$ The University of Sydney, NSW 2006, Australia \\ ${ }^{\dagger}$ The University of Technology Sydney, NSW 2007, Australia \\ Email:(samir.gautam; yuezhu.lu; weidong.xiao)@sydney.edu.au; Dylan.Lu@uts.edu.au
}

\begin{abstract}
Single phase grid connected converters in Photo$\operatorname{voltaic}(\mathbf{P V})$ and Electric Vehicles(EV) are finding their application in reactive power support to grid. A power feedback is generally integrated in the control loop for this purpose. Fast and accurate calculation of active and reactive power of the converter is necessary for swift response of controller in regulating output power. This also prevents converter from being overloaded. This paper presents a fast and simple technique to measure the active and reactive power component utilizing wave-shape similarity of sine and cosine function. The performance of the proposed method is compared with dq transformation based approach. The discussions and simulations show superiority of the proposed method in dynamics and implementation.
\end{abstract}

Index Terms-Reactive Current Component, DQ SRF, Orthogonal Signal Generation

\section{INTRODUCTION}

Lately, grid connected converters in PV and electric vehicles are being considered for applications beyond their primary functions. They have potential to provide reactive support to grid on demand to address power quality issues [1-3]. The reactive power these converters can provide, are limited by the rated power processing capability of semiconductors and passive components. To effectively realize this support, without overburdening the converters, fast and accurate computation of instantaneous active and reactive power of the converter is of paramount importance. This also aids in feedback control system for regulation of active and reactive component. There are two approaches to control active and reactive power, one through changing converter current magnitude and phase [3-4], and other by governing power angle and converter output voltage [5]. The former approach provides ease of implementation. A general control structure facilitating decoupled power control of single phase grid connected converter is shown in Fig. 1. Grid voltage is usually a known and uncontrollable parameter, and using it we can calculate active and reactive power, provided active and reactive components of converter current are known. To regulate these components independently, the key is calculating converter power components, determining reference current and converter current control. The power calculation unit, responsible for disintegrating current into active and reactive component is highlighted in the figure. The reference current is determined based on error between detected component and reference set points for each power component.

Two things are decisive in current detection, accuracy and the

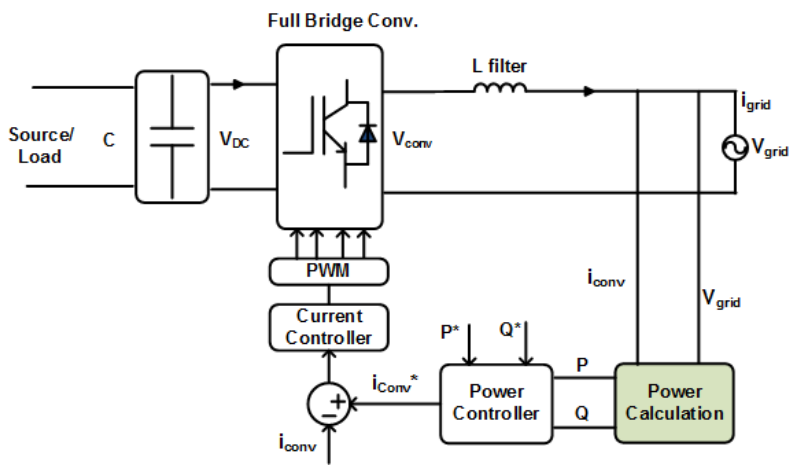

Fig. 1. Overall control structure for single phase grid connected converter

dynamics, any defect here will result in inappropriate control of converter current. The reference current extraction procedure is generally based on either time domain or frequency domain methods, with former preferred for their real-time acquisition. The delay and slow response of frequency domain methods are non-desirable. A simple method is multiplying grid voltage and converter current, to get instantaneous power. This, however, contains variations at twice the grid frequency, thus necessitating a Low Pass Filter (LPF) with low cut off frequency [6]. Despite its simplicity, the slow transient response induced by filter is the biggest drawback. Inspired from Instantaneous Reactive Power Theory (IRPT) and Synchronous Reference Frame(SRF) theory for three phase system, their application has been extended to single phase system as single phase pq, dq-SRF methods [7-8]. These methods require Orthogonal Signal Generation (OSG) to emulate two orthogonal signals in reference frame. To realize OSG, Time delay approach, Hilbert Transform, Second Order Generalized Integrator or All Pass Filter is used [9-10]. PQ method is sensitive to grid voltage distortion while $\mathrm{DQ}$ method requires synchronization signal as an extra requirement. DQ SRF is based on park transformation, where the fundamental active and reactive component of the converter current are converted to DC quantities on rotating frame. For distorted current, additional LPF must be used in the rotating frame to extract these DC quantities. Fig. 2 shows the DQ SRF method for single phase system. The OSG structure introduces at least a quarter of cycle delay and if LPF is present in the DQ frame for non-ideal case, the transient response further deteriorates 


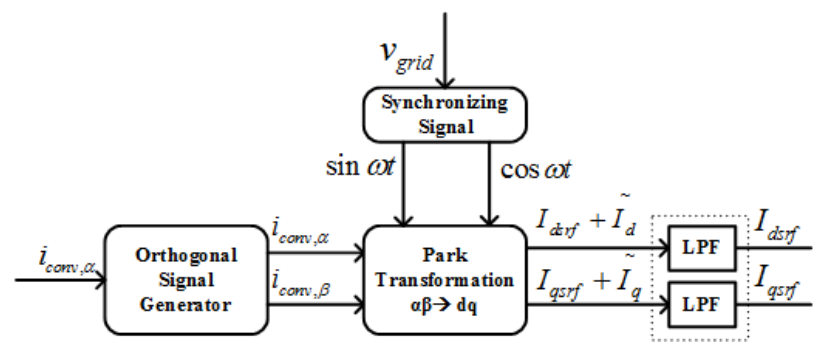

Fig. 2. SRF based dq method for single phase system

depending on filtering operation. The crucial issue however is how to obtain the orthogonal signal fast and accurately. The authors in [11] proposed an improved OSG structure using derivative operation. This gives instantaneous generation of orthogonal component but is prone to system instability in rapid current change.

There have been other techniques which have bypassed PQ and DQ methods. The article in [12] presents a simple yet powerful method to calculate the active and reactive component of the current with 2 LPF and 4 multipliers. However, the dynamics is still dictated by the LPFs characteristics and to eliminate double frequency components, lower cut-off frequency of LPF slows down the transient. A simple technique based on trigonometric orthogonal function is presented in [13], with a structure similar to [12], but with Moving Average Filter instead of LPF. It still requires a fundamental cycle for all transients to vanish. Authors in [14] use two sampled current in one cycle to calculate active and reactive component, but still has an inherent fundamental cycle delay. A power calculation method based on adaptive filtering is introduced in [15] with a settling time of $5 \mathrm{~ms}$, but it is more intricate than the previous stated techniques. Though complexity could be of least concern with the control and signal processing being performed in high end processors, still the simple implementation gives an added worth. The reactive current extraction for single phase system continues to be of significant research interest. This paper presents a simple and fast instantaneous reactive current detection for grid connected converters. The proposed structure uses two phase delay units, two multipliers and four summations, while it can reach stable value within $1 / 8$ th of fundamental cycle.

\section{Proposed Current Detection Method}

The general structure of a single-phase grid connected converter is shown in Fig. 1. The instantaneous grid voltage and converter current considering only fundamental component is defined as:

$$
\begin{gathered}
v_{g}=V_{m} \sin \left(\omega_{1} t\right) \\
i_{c o n v}(t)=I_{1} \sin \left(\omega_{1} t-\phi_{1}\right) \\
=I_{1}\left[\sin \left(\omega_{1} t\right) \cos \phi_{1}-\cos \left(\omega_{1} t\right) \sin \phi_{1}\right]
\end{gathered}
$$

where $V_{m}$ and $I_{1}$ are peak values of grid voltage and converter current respectively, while $\phi_{1}$ is the phase shift between them. $\omega_{1}=2 \pi f_{\text {grid }}$ is the fundamental angular frequency of the grid voltage. The converter current has two components embedded in it, responsible for active and reactive power flow. The main aim is to extract these components. Two orthogonal synchronizing signals from grid voltage can be obtained through Phase Locked Loop (PLL) sub-system which are generally an inherent part of the grid connected converter control [10].

$$
\begin{aligned}
& v_{d}=\sin \left(\omega_{1} t\right) \\
& v_{q}=\cos \left(\omega_{1} t\right)
\end{aligned}
$$

Now, multiplying converter current with these synchronizing signal, two components, at DC and at $2 \omega_{1}$ is obtained, as in the case of amplitude modulation. The result of product with in-phase component gives, active component at DC, while the other gives reactive component at DC.

$$
\begin{aligned}
& i_{d}(t)=v_{d}(t) * i_{c o n v}(t) \\
& =\frac{I_{1}}{2}\left[\cos \left(\phi_{1}\right)-\cos \left(2 \omega_{1} t-\phi_{1}\right)\right] \\
& i_{q}(t)=v_{q}(t) * i_{c o n v}(t) \\
& =\frac{-I_{1}}{2}\left[\sin \left(\phi_{1}\right)-\sin \left(2 \omega_{1} t-\phi_{1}\right)\right]
\end{aligned}
$$

The DC component can be extracted using a LPF, but it will degrade the dynamic response. Using a Moving Average Filter (MAF) or Delayed Signal Cancellation(DSC) can eliminate double frequency component, but either way it would need at least half the period for transient to die off. The sine and cosine have same wave shape but are shifted $90^{\circ}$ apart. Delaying one of them by quarter of cycle and subtracting from other will result in self cancellation. We utilize this inherent symmetry, to phase shift $i_{d}$ and $i_{q}$ by $90^{\circ}$, equivalently delay of $1 / 8$ of grid voltage fundamental period, T. Apparently, the DC component remains unaffected by this operation.

$$
\begin{aligned}
& i_{d}{ }^{\prime}(t)=\left(i_{d}(t)-\pi / 2\right)=i_{d}(t-T / 8) \\
& =\frac{I_{1}}{2}\left[\cos \phi_{1}-\sin \left(2 \omega_{1} t-\phi_{1}\right)\right] \\
& i_{q}{ }^{\prime}(t)=\left(i_{q}(t)-\pi / 2\right)=i_{q}(t-T / 8) \\
& =\frac{-I_{1}}{2}\left[\sin \phi_{1}+\cos \left(2 \omega_{1} t-\phi_{1}\right)\right]
\end{aligned}
$$

The transfer function of the phase delay unit with a delay of $T_{d}$ is given in (8) and the bode plot is given in Fig.3

$$
P(s)=e^{-s T_{d}}
$$

Considering expressions (4-7), we can obtain active and reactive component through simple manipulation.

$$
\begin{aligned}
& I_{d}=\left(i_{d}(t)-i_{q}{ }^{\prime}(t)\right)+\left(i_{d}{ }^{\prime}(t)+i_{q}(t)\right) \\
& I_{q}=\left(i_{q}{ }^{\prime}(t)-i_{d}(t)\right)+\left(i_{d}{ }^{\prime}(t)+i_{q}(t)\right)
\end{aligned}
$$

The overall structure to realize the calculation of the active and reactive current component is shown in Fig. 4. The simplicity of the proposed method lies in obtaining the desired results with small number of calculations. 


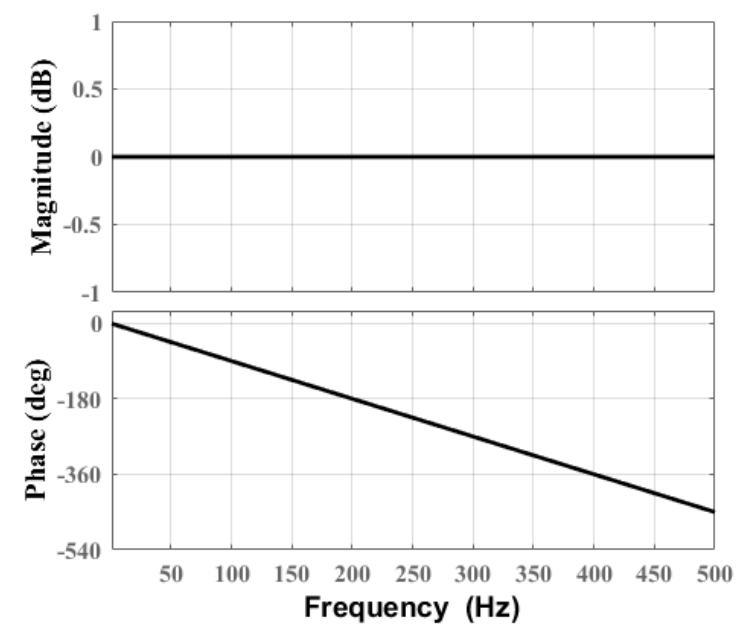

Fig. 3. Bode plot of the time delay unit

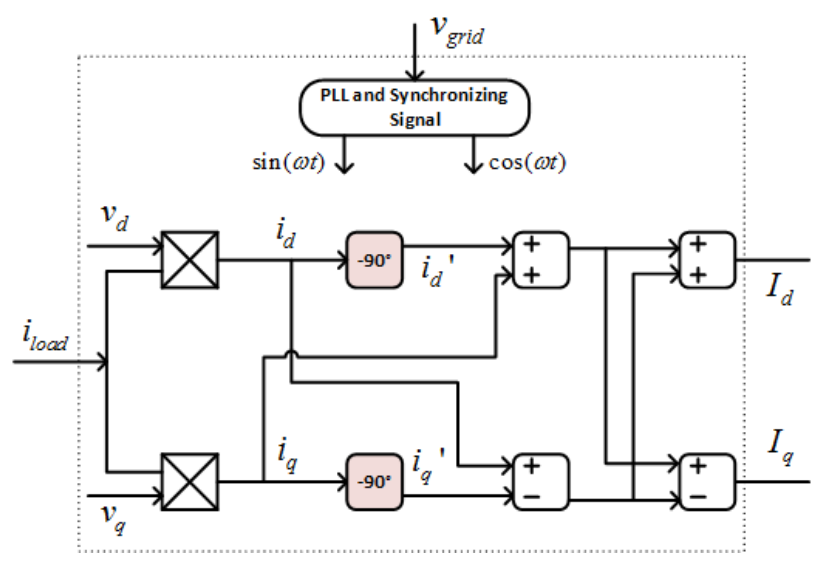

Fig. 4. Block diagram of the proposed current detection method

TABLE I

SiMULATION PARAMETERS

\begin{tabular}{|l|l|l|}
\hline Parameter & Symbol & Value \\
\hline Grid Voltage & $V_{\text {grid }}$ & $220 \mathrm{~V}$ RMS \\
\hline Grid Frequency & $f_{\text {grid }}$ & $50 \mathrm{~Hz}$ \\
\hline Inverter Power & $S_{\text {inv }}$ & $2.5 \mathrm{KVA}$ \\
\hline Grid side resistance & $R_{g}$ & $10 \mathrm{~m} \Omega$ \\
\hline
\end{tabular}

\section{COMPARISON AND SIMUlation VERIFICATION}

To verify the working of the proposed method, a simulation study is carried out in MATLAB/Simulink. Its performance is compared with the DQ SRF method, one of the mostly addressed approach in literatures. The proposed method is implemented in single phase grid connected voltage source inverter with reactive support. The parameters used are listed in Table 1. The VSI is considered ideal and modeled with a controlled current source capable of injecting the reference current exactly and instantaneously into the grid. The reference is calculated from active and reactive component, simulating an independent control of each.
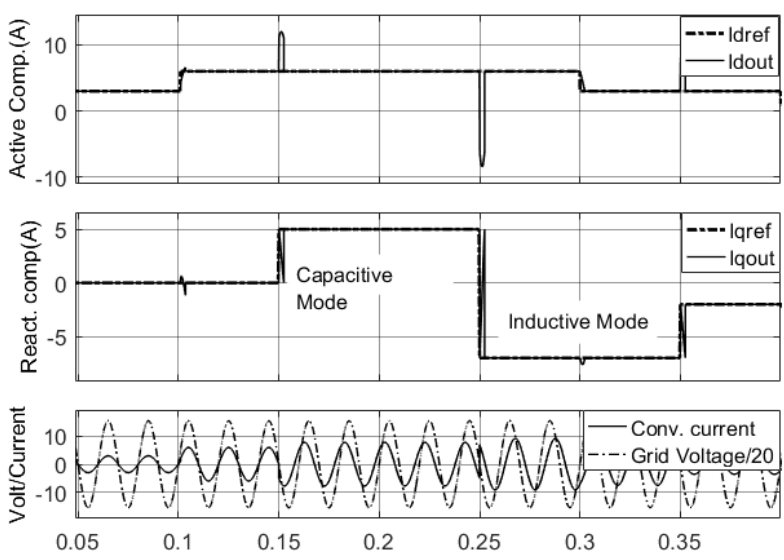

Fig. 5. Active and reactive component variation of converter current $\left(i_{\text {out }}\right)$

$$
\begin{aligned}
& I_{\text {out }}=\mathrm{I}_{m} \sin (\omega t-\phi) \\
& \mathrm{I}_{m}=\sqrt{I_{\text {dref }}^{2}+I_{\text {qref }}^{2}} \& \phi=\tan ^{-1}\left(\frac{-I_{\text {qref }}}{I_{\text {dref }}}\right)
\end{aligned}
$$

The active $\left(I d_{r e f}\right)$ and reactive $\left(I q_{r e f}\right)$ current reference to the converter is calculated as in:

$$
I_{d, r e f}=\frac{2 P}{V_{d}} \text { and } I_{q, r e f}=-\frac{2 Q_{r e f}}{V_{d}}
$$

where, $V_{d}$ is the active component, of the grid voltage in dq $\mathrm{SRF}$, which is equal to peak value for a perfectly synchronized frame. The synchronizing signals, which is in phase $\left(v_{d}\right)$ and quadrature $\left(v_{q}\right)$ with the grid voltage is obtained through PLL required for the current detection mechanism. Fig. 5 depicts the injection and detection of different amount of active and reactive component into the grid. The positive reactive component, correspondingly refers to capacitive mode by injecting reactive power, while negative reactive component corresponds to consuming reactive power from the grid. The measured component of the converter output $i d_{\text {out }}$ and $i q_{\text {out }}$ shows close resemblance with the reference component except at the transient state validating the accuracy of the proposed method.

Simulation results in Figs. 6 and 7 illustrate the instantaneous detection of current components during the transient state, as compared with the conventional dq SRF method. In SRF dq method, single-phase quantity is first converted to two orthogonal signals to form reference frame. This is converted to rotating frame using the transformation matrix constituted from synchronizing signal and given below:

$$
\left[\begin{array}{c}
i_{d s r f} \\
i_{q s r f}
\end{array}\right]=\left[\begin{array}{cc}
\sin \omega t & -\cos \omega t \\
\cos \omega t & \sin \omega t
\end{array}\right]\left[\begin{array}{l}
i_{\alpha} \\
i_{\beta}
\end{array}\right]
$$

In Fig. 6 the transient state is started at $t=0.101$, when the active power reference changes from $466.5 \mathrm{~W}$ to $933 \mathrm{~W}$. Similarly, in Fig 7, the transition of reactive power occurs from $0 V A R$ to $775 V A R$ at $t=0.15 s$. In both cases, the proposed method can settle to its steady state value precisely in $2.5 \mathrm{~ms}$, while dq-SRF takes around $5 \mathrm{~ms}$. The time required for measuring the change of converter current, in either case is dependent on OSG structure in dq SRF and phase delay 

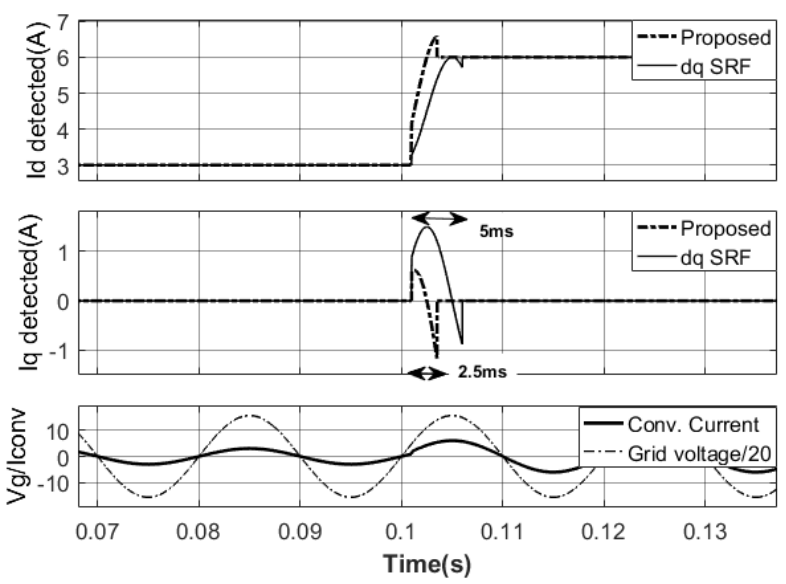

Fig. 6. Transient performance of two methods for active reference change
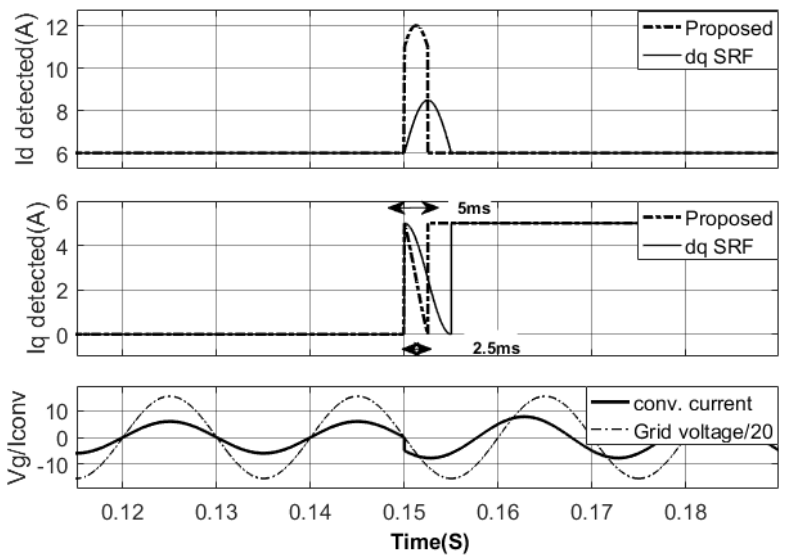

Fig. 7. Transient performance of two methods for reactive reference change

unit in proposed method. The decreased response time owes to presence of OSG structure in second harmonic frame. The figures 6-7 also demonstrate, the injected sinusoidal output current with respect to the down scaled grid voltage. An overshoot and undershoot is observed during the current component detection, the reason for this is mentioned in the Section IV. These simulation results establish the superiority of the proposed method in fast transient response.

Furthermore, the dq SRF method requires, four multipliers, two summation and one phase shift $(T / 4)$ delay operator, while the proposed method requires two multipliers, four summations and two phase shift $(T / 8)$ delay operators. Multiplication operation are more calculation intensive compared to addition, hence the proposed method presents comparatively lower implementation burden. The halving of transient duration can be attributed to location of OSG block in proposed method $\left(2 f_{\text {grid }}\right)$ as compared to in $\mathrm{DQ} \operatorname{SRF}\left(f_{\text {grid }}\right)$.

\section{IMPLEMENTATION CONSIDERATIONS}

\section{A. Phase Unbalance}

The $90^{\circ}$ phase delay at $2 \omega_{1}$ is equivalent to time delay $\left(T_{d}\right)$ of $2.5 \mathrm{~ms}$ for grid frequency of $50 \mathrm{~Hz}$. In discrete time, it can

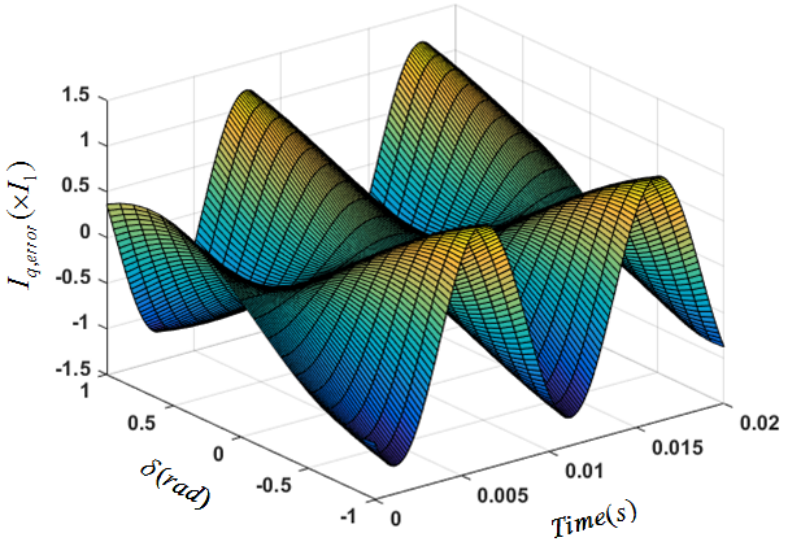

Fig. 8. The error in detected reactive current component due to phase error

be implemented as a delay of $\mathrm{N}$ sample points, which depends on the sampling frequency $f_{s}$.

$$
\begin{gathered}
d(z)=z^{-N} \\
N=\frac{T_{d}}{T_{s}}
\end{gathered}
$$

This can be practically realized using linear/circular buffer of length $\mathrm{N}$, storing $\mathrm{N}$ samples and operating in First in First Out method. Phase delay block is sensitive to the grid frequency. The grid frequency may vary within a specified range of $\pm 2 \mathrm{~Hz}[16]$ and accordingly, the phase delay block must adapt to the grid frequency, to realize exact 90-degree phase shift. The phase unbalance can cause an error in the detected current components.

Assume, the phase shift from the phase delay block be $(\pi / 2) \pm 2 \delta$. Where, $\delta$ is the steady state phase error due to the variation of grid frequency $(\omega)$ from its nominal value $\left(\omega_{1}\right)$.

$$
\delta=\left(\omega-\omega_{1}\right) \cdot T_{d}=\frac{\Delta \omega N}{f_{\text {sam }}} \mathrm{rad}
$$

The phase shifted/orthogonal components become

$$
\begin{aligned}
& i_{d}{ }^{\prime}(t)=\frac{1}{2} I_{1}\left[\cos \phi_{1}-\sin \left(2 \omega t-\phi_{1}+2 \delta\right)\right] \\
& i_{q}{ }^{\prime}(t)=\frac{1}{2} I_{1}\left[\sin \phi_{1}-\cos \left(2 \omega t-\phi_{1}+2 \delta\right)\right]
\end{aligned}
$$

Substituting above equations in equation (9-10) and undergoing trigonometric simplification, the following active and reactive components $(19,20)$ are deduced.

$$
I_{d}=I_{1} \cos \phi_{1}-\sqrt{2} I_{1} \sin (\delta)\left\{\cos \left(2 \omega t-\left(\phi_{1}-\delta+45\right)\right\}\right.
$$

$$
I_{q}=I_{1} \sin \phi_{1}-\sqrt{2} I_{1} \sin (\delta)\left\{\sin \left(2 \omega t-\left(\phi_{1}-\delta+45\right)\right\}\right.
$$

An error component at double line frequency, whose amplitude and phase are function of amount of unbalance, is 
mixed with the actual DC components, as shown in Fig. 7 (for $\phi_{1}=30^{\circ}$ ). Hence, it is necessary to adjust the delay time as per the change in grid frequency. This can be achieved by either updating the buffer length $\mathrm{N}$ or varying the sampling frequency. In this paper, we consider sampling frequency as constant hence an updated buffer length is calculated as:

$$
N=\operatorname{Round}\left(f_{s} / 8 * f_{g}\right)
$$

The small error due to rounding off non-integer $\mathrm{N}$ can be considered negligible for higher sampling frequencies and is beyond the scope of this paper.

\section{B. Transient Response}

The summation and multiplication are real time operations and follow the input changes instantaneously. The response of the detection mechanism is thus decided by the phase delay unit. Let us assume, the input current changes from $I_{1} \sin \left(\omega t-\phi_{1}\right)$ to $I_{1}^{\prime} \sin \left(\omega t-\phi_{1}^{\prime}\right)$, at $t=t 1$ then the instantaneous components in eqn (4-5) become:

$$
\begin{aligned}
i_{d}(t) & =\frac{1}{2} I_{1}{ }^{\prime}\left[\cos \left(\phi_{1}{ }^{\prime}\right)-\cos \left(2 \omega_{1} t-\phi_{1}{ }^{\prime}\right)\right] \\
i_{q}(t) & =\frac{1}{2} I_{1}{ }^{\prime}\left[-\sin \left(\phi_{1}{ }^{\prime}\right)+\sin \left(2 \omega_{1} t-\phi_{1}{ }^{\prime}\right)\right]
\end{aligned}
$$

While the delayed signal $(6-7)$ still has the old values as below, for $(t 1+T / 8)$ :

$$
\begin{gathered}
i_{d}(t)^{\prime}=\frac{1}{2} I_{1}\left[\cos \phi_{1}-\sin \left(2 \omega_{1} t-\phi_{1}\right)\right] \\
i_{q}(t)^{\prime}=\frac{1}{2} I_{1}\left[-\sin \phi_{1}-\cos \left(2 \omega_{1} t-\phi_{1}\right)\right]
\end{gathered}
$$

The active and reactive component, changes $I d$ and $I q$ be:

$$
\begin{gathered}
I_{d}{ }^{\prime}=I_{1}{ }^{\prime} \cos \phi_{1}{ }^{\prime} \& I_{q}{ }^{\prime}=-I_{1}{ }^{\prime} \sin \phi_{1}{ }^{\prime} \\
I_{d}=I_{1} \cos \phi_{1} \& I_{q}=-I_{1} \sin \phi_{1} \\
\Delta I_{d}=I_{d}{ }^{\prime}-I_{d} \\
\Delta I_{q}=I_{q}{ }^{\prime}-I_{q}
\end{gathered}
$$

The instantaneous detected current component, during this transient period, using the equation (9-10) and trigonometrical identities results in eqn(28-29):

$$
\begin{aligned}
I_{d}(t)=\frac{\left(I_{d}{ }^{\prime}+I_{d}\right)+\Delta \mathrm{I}_{\mathrm{q}}}{2} & +\left(\frac{\Delta I_{d}+\Delta I_{q}}{2}\right) \sin (2 \omega t) \\
& -\left(\frac{\Delta I_{d}-\Delta I_{q}}{2}\right) \cos (2 \omega t) \\
I_{q}(t)=\frac{\left(I_{q}{ }^{\prime}+I_{q}\right)-\Delta \mathrm{I}_{d}}{2}+ & \left(\frac{\Delta I_{d}-\Delta I_{q}}{2}\right) \sin (2 \omega t) \\
& +\left(\frac{\Delta I_{d}+\Delta I_{q}}{2}\right) \cos (2 \omega t)
\end{aligned}
$$

The active and reactive component during the transient depends on the amount of the change in both component, and will last for $T / 8$.
Applying similar transient analysis for dq SRF frame method given in eqn(13),(30-31)is obtained

$I_{d s r f}(t)=\frac{I_{d}+I_{d}{ }^{\prime}}{2}-\left\{\frac{\Delta I_{d}}{2}\right\} \cos (2 \omega t)+\left\{\frac{\Delta I_{q}}{2}\right\} \sin (2 \omega t)$

$I_{q s r f}(t)=\frac{\left(I_{q}{ }^{\prime}+I_{q}\right)}{2}+\left\{\frac{\Delta I_{d}}{2}\right\} \sin (2 \omega t)+\left\{\frac{\Delta I_{q}}{2}\right\} \cos (2 \omega t)$

Since the active and reactive components are coupled with each other, the deviation in one of them will affect the other component. Two cases are studied, to analyze effect of changing component on detection of fixed component.

Case $I: \Delta I_{d}=0$

Here only the reactive component undergoes the change, with active component remaining same. The detected active component by proposed method and dq SRF from eqn (28)\& (30) for the transient duration $(t 1<t<T / 8$ and $t 1<t<T / 4)$ is calculated to be:

$$
\begin{gathered}
I_{d}(t)=I_{d}{ }^{\prime}+\frac{\Delta \mathrm{I}_{\mathrm{q}}}{2}+\frac{\Delta I_{q}}{\sqrt{2}} \cos \left(2 \omega t-45^{\circ}\right) \\
I_{d s r f}(t)=I_{d}{ }^{\prime}+\frac{\Delta I_{q}}{2} \sin (2 \omega t)
\end{gathered}
$$

Case $I I: \Delta I_{q}=0$

Consider, only active component varies keeping reactive component equal. The detected reactive component during the transient for the proposed method and dq SRF will result in:

$$
\begin{gathered}
I_{q}(t)=I_{q}{ }^{\prime}-\frac{\Delta \mathrm{I}_{d}}{2}+\frac{\Delta \mathrm{I}_{d}}{\sqrt{2}} \cos \left(2 \omega t-45^{\circ}\right) \\
I_{q s r f}(t)=I_{q}{ }^{\prime}+\frac{\Delta I_{d}}{2} \sin (2 \omega t)
\end{gathered}
$$

It can be seen there is an additional dc component mixed with the actual component during the transient period in the proposed method. Additionally, the superimposed double harmonic component is $70 \%$ of the changes in proposed method, while it is only $50 \%$ in case of the dq SRF. Thus, the proposed method may present larger overshoot and undershoot. This depends on also the time when the transient occurs, which determines which part of the sinusoid is superimposed. A saturation block can be enforced to prevent the detected component exceeding rated values. Besides, the transient only lasts for small amount time of $T / 8$, which is half of DQ SRF.

\section{CONCLUDing REMARKS}

This paper presented a simple active and reactive component detection mechanism based on the intrinsic property presented by wave-shape symmetry of sine and cosine waves. The proposed method can calculate the active and reactive current component in $1 / 8$ th of the fundamental period, which is equivalent to the time delay introduced by the $90^{\circ}$ phase delay block. The simulation results and discussion validated the working of the proposed current detection mechanism. Future work will include extending the application where 
distorted current is present as in active power filtering, detailed transient response analysis and experimental verification.

\section{ACKNOWLEDGMENT}

The authors would like to acknowledge the financial support provided by the University of Sydney International Scholarship (USydIS) for this research work.

\section{REFERENCES}

[1] M. Falahi, H.-M. Chou, M. Ehsani, L. Xie, and K. L. Butler-Purry, "Potential power quality benefits of electric vehicles," IEEE Transactions on sustainable energy, vol. 4, pp. 1016-1023, 2013.

[2] K. Turitsyn, P. Sulc, S. Backhaus, and M. Chertkov, "Options for control of reactive power by distributed photovoltaic generators," Proceedings of the IEEE, vol. 99, pp. 1063-1073, 2011.

[3] K. Knezovi, M. Marinelli, R. Juul, P. B. Andersen, C. Trholt, and F. Sossan, "Analysis of voltage support by electric vehicles and photovoltaic in a real Danish low voltage network," in Power Engineering Conference (UPEC), 2014 49th International Universities, 2014, pp. 1-6.

[4] F. El Aamri, H. Maker, A. Mouhsen, and M. Harmouchi, "A new strategy to control the active and reactive power for single phase grid-connected PV inverter," in Renewable and Sustainable Energy Conference (IRSEC), 2015 3rd International, 2015, pp. 1-6.

[5] T. C. Binh, M. T. Dat, N. M. Dung, P. Q. An, P. D. Truc, and N. H. Phuc, "Active and reactive power controller for single-phase grid-connected photovoltaic systems," in Proceedings of AUN-SEEDNet Conference on Renewable Energy, Bandung, Indonesia, 2009.

[6] E. T. Andrade, P. E. M. Ribeiro, J. O. P. Pinto, C.-L. Chen, J.-S. Lai, and N. Kees, "A novel power calculation method for droop-control microgrid systems," in Applied Power Electronics Conference and Exposition (APEC), 2012 Twenty-Seventh Annual IEEE, 2012, pp. 2254-2258.

[7] M. T. Haque, "Single-phase pq theory for active filters," in TENCON'02. Proceedings. 2002 IEEE Region 10 Conference on Computers, Communications, Control and Power Engineering, 2002, pp. 1941-1944.

[8] S. Gautam, P. Yunqing, Y. Kafle, M. Kashif, and S. U. Hasan, "Evaluation of Fundamental dq Synchronous Reference Frame Harmonic Detection Method for Single Phase Shunt Active Power Filter,' International Journal of Power Electronics and Drive Systems, vol. 4, p. 112, 2014.

[9] Y. F. Wang and Y. W. Li, "A grid fundamental and harmonic component detection method for single-phase systems," IEEE Transactions on Power Electronics, vol. 28, pp. 2204-2213, 2013.

[10] S. Gautam, P. Yunqing, M. Kashif, Y. R. Kafle, Z. Z. Hua, and L. Bo, "Study on software phase locked loop for single phase active power filter," 2013.

[11] A. Luo, Y. Chen, Z. Shuai, and C. Tu, "An improved reactive current detection and power control method for single-phase photovoltaic gridconnected DG system," IEEE Transactions on Energy Conversion, vol. 28 , pp. 823-831, 2013.

[12] T. Tanaka, E. Hiraki, N. Ishikura, Y. Omura, and M. Yamamoto, "A novel real-time detection method of active and reactive currents for single-phase active power filters," in Power Electronics Specialists Conference, 2007. PESC 2007. IEEE, 2007, pp. 2933-2938.

[13] H. Yi, F. Zhuo, F. Wang, Y. Li, and Z. Wang, "A Single-phase Harmonics Extraction Algorithm Based on the Principle of Trigonometric Orthogonal Functions," JOURNAL OF POWER ELECTRONICS, vol. 17, pp. 253261, 2017.

[14] C.-H. Chang, Y.-H. Lin, Y.-M. Chen, and Y.-R. Chang, "Simplified reactive power control for single-phase grid-connected photovoltaic inverters," IEEE Transactions on Industrial Electronics, vol. 61, pp. 22862296, 2014.

[15] Y. Yang and F. Blaabjerg, "A new power calculation method for singlephase grid-connected systems," in Industrial Electronics (ISIE), 2013 IEEE International Symposium on, 2013, pp. 1-6.

[16] I. Carugati, P. Donato, S. Maestri, D. Carrica, and M. Benedetti, "Frequency adaptive PLL for polluted single-phase grids," IEEE Transactions on Power Electronics, vol. 27, pp. 2396-2404, 2012. 